\title{
Study on College Information Resource Sharing under the Environment of Cloud Computing
}

\author{
Wen-Jing YANG ${ }^{1, a}$, Hai-Yan $Z_{H A O}^{1, b^{*}}$, Jin MENG ${ }^{1, c}$, Xiao-Shuang WANG ${ }^{1, c}$ \\ and $\mathrm{Yi} \mathrm{HE}^{1, \mathrm{C}}$ \\ ${ }^{1}$ Xi'an Communication Institute, Xi'an 710106, China \\ a51375908@qq.com, b813086903@qq.com, 583822272@qq.com \\ ${ }^{*}$ Corresponding author
}

Keywords: Information Resource, Cloud Computing, College.

\begin{abstract}
Combined with cloud computing technology, we described its role and advantages in resources building of the university. We initially developed a resources sharing platform based on cloud. The strategy of the developments of standardized college information resources, the data central of cloud computing, the unification of college information resources and the sharing platform were discussed. The availability for implements were analyzed, aiming at giving useful information to college information resources sharing.
\end{abstract}

\section{Introduction}

With the popularization and application of information technology, information resource development achievements are significant in Information Resources of Universities. On the basis of the surge in the number of information resources, the construction is developing in the towards of diversified content, forms, complex direction. Being faced with the complex mass of information resources to effectively improve service quality, playing the maximum efficiency of resources have higher requirements for deep resources development and utilization. Cloud computing, as a new method of shared infrastructure, has been promised in the construction and application of information resources in colleges.

\section{Advantages of Cloud Computing in Promoting the College Resources Sharing}

Virtualization technology improves the utilization of basic facilities in colleges. Virtualization technology, as one of the core technology of cloud computing, puts the universities existing hardware devices into a virtual resource pool, masking the differences between the physical device servers, networks, storage, dynamic deployment, and assigning to different applications and services, which improve the utilization and lower construction costs of underlying resource. Experiments show that the use of virtualization technology enables the average utilization of each server to increased from $7 \%$ to $68 \%$ to $80 \%$; reduce the deployment time from hours to minutes; rebuilt server and application load times from $20 \mathrm{~h} \sim 40 \mathrm{~h}$, being reduced to $15 \mathrm{~min} \sim 30 \mathrm{~min}$; construction cost savings are more than $30 \%[1]$.

Cloud storage technology improves the information sharing capability of colleges. Heterogeneous information resources of the physical location of universities are integrated, decentralized and stored in the "cloud" massive computing and storage cluster, the cloud centralized management and scheduling[2]. Distribution of information resources and centralized storage management and information resources avoids duplication, eliminates information silos, and creates a common information sharing space.

Parallel processing and distributed computing technology offers powerful computing capabilities and dynamic scalability. Through large-scale distributed file system, the way information resources to block and multiple-copy backups are stored in the cloud disperses. The analysis of the data processing are also undergoing large-scale parallel computing and distributed computing technology. In addition, the cloud platform provides a variety of open API supports 
heterogeneous access, having flexibility to expand according to the demands, automatic synchronization, real-time updates of information resources and create conditions for the sharing of information resources in a wider range[3].

Cloud computing creates a stable and secure information space. Through the cloud computing, we can achieves distributed, heterogeneous information resources load balancing and avoid the network overload. The use of redundancy, fault tolerance, disaster recovery and other technology protects against data loss caused by improper operation, disruption and viruses, service interruptions, hardware damage and other threats to college information resources sharing issues. It also frees users from complex, highly specialized network security and system operation and maintenance, and focus on the resources of the application itself[4].

\section{College information resource sharing policy in cloud computing environment}

Information resources of university combines with the problems and benefits of cloud computing in the resources of construction. Here, we intend to use cloud computing technology to build cloud college information resource sharing platform, which is shown in Figure 1. This mode is calculated by the college of information resources in cloud environments, which consists of sharing strategies, mechanisms to analyze the feasibility of cloud model and parts of the cloud model selection. Under a cloud computing environment to college information resource sharing strategy of focus, from the College of Information Resources standardization, we can set up cloud computing data centers, optimize the integration of information resources and build colleges information sharing and other aspects of the cloud platforms.

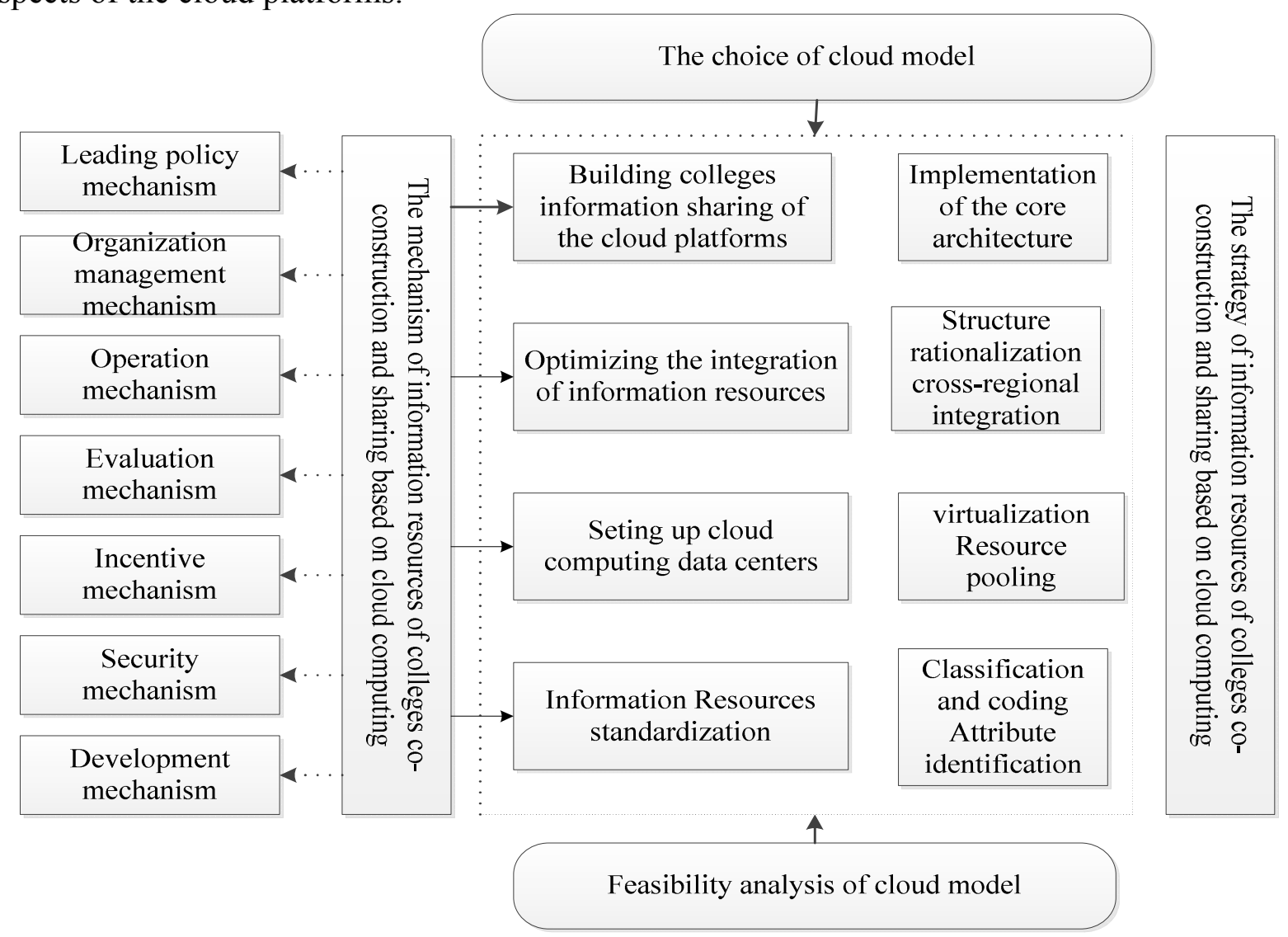

Fig. 1 Information resource sharing model for colleges in cloud computing environment

Standardization of information resources of colleges in order to lay the foundation for resource sharing. In the process of achieving the general college information resources sharing, we should also focus on domestic and international reference for existing information resources and to strictly implement construction standards, regulate the types and properties of colleges of information resources, in order to establish a unified information resource construction standards. Therefore, we can solve heterogeneous repositories and information silos issues and create conditions for the 
construction of general college information resource sharing platform. On this basis, full use of advanced information technology can make high-quality general college information resources can be shared in a wider range. Given that the current technology and standards at various colleges and universities to establish information resource library use is not unified, in order to ensure that the information resource sharing work can be carried out smoothly, heterogeneous information resources has become an indispensable step to standardize. College of information resources is a standardized in hierarchical evolutionary process. According to the existing resources construction standards in references at home and abroad, it can be standardized in two ways: classification and coding and attribute identifies. Classification code is from the macro to the classification of various information resources and give encoded value which is assigned to each attribute identifies an information resource from the micro. It should have a series of property value and ultimately the formation table metadata instance of various information resources is formed.

Building the cloud computing data centre. With the increasing popularity of information technology in the field of education, various information resources increasing, the size of the data center has been expanded, secure data center reliability of the higher requirements, the traditional data center for business continuity poor existence, long deployment cycles flexibility, poor operation and maintenance cost issues have become increasingly prominent. Using cloud computing and virtualization technology to create next-generation data center, we can achieve data center computing services, resource virtualization and management intelligence services in the college of information resources sharing. Cloud computing data centers with virtualization, elastic recovery, optimize collaboration, modular and other features, is gathered by a large number of high-performance storage device cluster, routing network equipment like clusters and server clusters, with virtualization, resource pooling, dynamic management and expansion technology to build more storage, networking, computing resource pool, intelligent management and deployment of resources, tasks, and to provide users with an application, parallel computing, large storage, programming tools, communications services, collaboration tools and other cloud services. Also, it enables a large number of complex parallel computing process to ensure safe and reliable data storage, improving the quality and efficiency of information resources and sharing.

Optimizing the integration of colleges and universities information resources and improving the resource utilization. On the one hand, we should promote the rationalization of the college of information resources structure. Information rationalize resource structure can ensure the optimum configuration of information resources, avoid duplication of resources and maximize resource sharing. From information resource construction of college to college information resource for the study, as a way to optimize the integration of the latest achievements of information technology and as a mean to build a shared purpose, from the space of the college of information resources rational distribution, we can form a high quality, multi-level, comprehensive, service-oriented information resource system of college. Various colleges and universities based on the school characteristic information resource should be organized, including figure, text, sound, and other information resources like orderly organization, optimization, forming the characteristic media library, online courses and other resources library full of information resources of the largest colleges utility. On the other hand, we should focus on integrating cross-regional information resources in colleges and universities. All colleges and universities in terms of resource development, should actively participate in and promote the formation of a regional center of the college of information resources sharing alliance. We can build a regional information sharing network for colleges and actively participate in regional integration and the unity of digital resources division of labor, and actively develop its own characteristics, in accordance with professional information resource library school personnel training objectives, and with the cloud computing technology integration in the region each colleges original information resources. The aim is to build common college information resources sharing in the cloud computing environment platform. Thus, creating a favorable space for the College to share resources in the network environment is an effective way to maximize the sharing of information resources colleges implementation. 
Construction on college information resource sharing cloud platform and achieving full sharing of resources. Cloud computing is a new resource sharing, management and service delivery model. Its core concept is the integration of a large number of scattered, isolated information resources, establishment of a unified management of the resource pool to meet the individual needs for users. Its aim is to achieve full sharing of resources, and its superior computing power, large memory, high availability, high scalability, using inexpensive and so has been widely applied in the fields of resource sharing, service and management. We can apply information resource construction of college to build a cloud platform sharing of existing information resources to integrate the various colleges, the cloud unified management and scheduling, service-oriented architecture and the types of information resources available on demand customization service delivered to users in the form of help, solving the information resource construction in colleges and universities, such as the existence of low resource utilization, information silos, data security and other issues. It can improve the general college information resources construction environment, general information resources and then enhance the quality of service and universities, colleges and promote the sharing of information resources. Construction of information resources with the current problems and colleges cloud computing architecture, service model and other related concepts, we can clearly establish a service-oriented architecture (SOA) and information resources.

Implementation of distributed storage and centralized management of information resources can achieve the open sharing of information resources, sharing colleges cloud platform. The platform has the physical layer, the platform layer, resource integration layer, application layer and five-layer architecture access layer, providing virtual physical resources through the logic, application development, operation, testing, database platform resources, and various specific functions application resources. The platform also has a user authentication, single sign-on and one-stop access.

\section{Feasibility Analysis}

Theoretical basis. On the one hand, there are plenty of recent theoretical results related to the construction of numerous foreign information resources. Construction of the advanced experience of foreign information resources in government decision-making, construction standards, organization and other aspects of capital investment provide a reference for the information resource construction of colleges. On the other hand, along with the maturing of cloud computing technology and extensive application of the relevant literature on the cloud computing, the market is getting rich. Cloud computing applies college information resource sharing and provides sufficient theoretical basis and practical guidance.

Technical Support. Many foreign IT companies such as Google, Amazon, Microsoft, IBM and Hewlett-Packard have put forward their own cloud initiatives, in the fields of government, business, education and other application. Cloud computing technology and its practical application have been widely carried out in all sectors of society and provide theoretical reference and technical support for the construction of general information resources sharing universities cloud-based computing.

Economic costs. First, the use of cloud computing technology can make a large number of low-cost ordinary computer integrate into high-performance computing centers and mass storage resource pools, improving performance computing and storage hardware environment in the original basis of the same. Infrastructure virtualization improves server, storage, network and other equipment resource utilization, cost and energy in order to create a green data center. With cloud storage to college information resource unified storage in the cloud, interconnected sharing of resources avoid duplication and waste resources caused by the human, material and financial resources. The complex platform maintenance, resource allocation, system monitoring, security management work to a professional cloud service providers that can complete and reduce operation and maintenance costs of information resources college. For users, there is no need for a terminal device with high-end data computing support, program operation and other tasks, and also no need for having to spend too much energy to install, upgrade and maintenance of software and hardware resources. What users need including computing resources, platforms and software applications environment can simply go 
through the browser customization, demand access, usage-based billing to create a fast, efficient, affordable, safe and stable information space.

\section{Summary}

This paper develops the information resources sharing mode based on cloud computing techniques and analyzes the availability of resources sharing among colleagues. This study aims to cloud computing technology in information resource of the college, in order to provide new ideas for resource sharing. Of course, to build a cloud platform information sharing, there are also challenges. Standardization of cloud computing has not been perfect. Cloud platforms supporting cloud service providers not yet reached unified technical and service standards. In service capabilities, quality of service, operation and maintenance, and energy management and other aspects of uneven, we also face to different data and applications that cannot be migrated to mutual cloud platform, resulting in isolated cloud platform and cloud services providers monopoly issues. Thus, to speed up the standardization of the cloud platform of cloud techniques in order to provide a unified standard interface and provide users with a unified standard cloud services, has become an urgent problem in the future.

\section{Acknowledgement}

This research was financially supported by The national social science fund(14GJ003-029).

\section{References}

[1] Virtualization and cloud computing group, Cloud computing technology and practice, third ed., Electronic industry publishing, Bei Jing, 2011.

[2] Yue LIU, Cloud computing technology and application, The ministry of industry and information technology telecommunications communication information research institute. 21(2009)4-6.

[3] Wanyun, Cloud computing technology, platform and application example, first ed.,Tsinghua University Press, Bei Jing, 2011.

[4] Jiang Xiaoxu, Luo Hui, Design plan of the college information resources sharing platform based on cloud computing, China Medical Education Technology. 27(2013)38-42. 